\title{
Visual-motor embodiment of language: a few implications for the neuropsychological evaluation (in Alzheimer's disease)
}

\author{
Éric Laurent ${ }^{1,2 *}$ and Nicolas Noiret ${ }^{1}$ \\ ${ }^{1}$ Laboratoire de Psychologie, Université de Franche-Comté, Université Bourgogne Franche-Comté, Besançon, France, \\ ${ }^{2}$ Maison des Sciences de l'Homme et de l'Environnement, Centre National de la Recherche Scientifique, Université de \\ Franche-Comté, Université de technologie Belfort-Montbéliard, Université Bourgogne Franche-Comté, Besançon, France
}

Keywords: vision disorders, embodied cognition, modularity of mind, enaction, enactivism, neuropsychological assessment, neuropsychological testing, sensory deficits

"The productive combination of adjectives, nouns, verbs, and other linguistic elements corresponds to the productive combination of perceptual symbols for properties, entities, processes, and other conceptual elements" (Barsalou, 1999, p. 594)

\section{Introduction}

For several decades, researchers in cognitive neuroscience and cognitive psychology have developed works concerning the close relationships between "lower-level" perceptual/motor and "higher-level" conceptual/linguistic processes (Harnad, 1987; Goldstone, 1994; Barsalou, 1999; Pulvermüller and Fadiga, 2010). Some of them suggested to "reunit" perception and conception (Goldstone and Barsalou, 1998), studied the interactions between language and action (Glenberg and Kaschak, 2002; see Pulvermüller and Fadiga, 2010, for a global picture in neuroscience), or were interested in the relationships between language and other bodily (emotional) states (Glenberg et al., 2005).

Manuel Menéndez-González, Hospital Álvarez-Buylla, Spain

Reviewed by:

Lynden K. Miles,

University of Aberdeen, UK

*Correspondence:

Éric Laurent,

eric.laurent@laurent-lab.com

Received: 21 July 2015 Accepted: 10 September 2015 Published: 30 September 2015

Citation:

Laurent É and Noiret N (2015)

Visual-motor embodiment of language: a few implications for the neuropsychological evaluation (in Alzheimer's disease).

Front. Aging Neurosci. 7:184. doi: 10.3389/fnagi.2015.00184 only does Alzheimer's Disease (AD) lead to alteration in brain cortical structure and cognitive impairment, but it also conducts to deep changes in both visual system organization and visionbased performances (Tzekov and Mullan, 2013).

We recommend that both the perceptual impairment found in $\mathrm{AD}$ and the interactions between lower-level and higher-level cognition be taken into account by neurospychologists in order to avoid misattribution of performance deficits.

We first mention a recent research concerning language evaluation in AD and discuss main limitations of modular evaluation in that type of context. Then, we present main features of the visual "function" impairment in $\mathrm{AD}$, the impacts of perceptual changes over higher-level cognition, and finally, we provide general recommendations for neuropsychological testing of higher-level cognitive "functions".

\section{Linguistic Evaluation in AD}

Drummond et al. (2015) reported an interesting research in which language production processes were evaluated in patients with $\mathrm{AD}$, amnestic mild cognitive impairment (a$\mathrm{MCI}$ ), and controls. In contrast with many neuropsychological tests aiming at evaluating language on the basis of simple concept production (e.g., naming), the authors developed 
a "narrative test", in which participants were supposed to narrate a story from a sequence of visually presented actions. Overall, the authors found that patients with a-MCI already presented narrative deficits in comparison with the control group. Interestingly, a-MCI discursive deficits were lower than those presented by patients with $\mathrm{AD}$, which may be interpreted as an intermediate level of deficiency between healthy elderly and patients with $\mathrm{AD}$.

The research is interesting and allows us to examine usual practices in neuropsychology and neuropsychological research. Although the participants in this kind of research generally undergo both neuropsychological and visual (i.e., acuity) assessments, the real involvement of language "function" in the deficits found in patients with AD or a-MCI can be questioned. As we will see later, $\mathrm{AD}$ can lead to several visual processing impairments that influence higher-level cognitive performance so that checking for normal or corrected-to-normal visual acuity is not sufficient to control for lower-level influence on cognitive performance. Typically, whether in neuropsychology or in speech therapy, language abilities in $\mathrm{AD}$ are often evaluated by tests involving the visual "function". For instance, in the naming tests, patients have to orally produce the word represented by a drawing picture. Similarly, oral comprehension tests ask patients to indicate, among several pictures, which corresponds to a word or a sentence read by the examiner. In other words, patients have to visually recognize a picture (as in the naming tests) based on an oral description. The matching category tests also require patients to choose-among several visual items - the one that is semantically associated with a target item. Finally, tests that focus on graphic abilities (e.g., dictation, free writing, writing description) also rely on visual "functions".

When performances are altered in the tests such as those described above, any earlier level of information processing can be involved (Greene, 2005). Although they are mainly employed to evaluate language, these tests can also reflect visual "function" impairment. The semantic recognition of drawings or pictures implies that patients rely on good visual acuity, color vision, contrast sensitivity, and oculomotor processing. Misinterpretations of $\mathrm{AD}$ patient troubles may arise if visual performances are not taken into account (and controlled for in statistical analyses).

\section{The Visual "Function" in AD}

If cognitive-and especially memory-disorders are a hallmark of $\mathrm{AD}$, it is less widely known but well established, that many visual processes are also altered at multiple levels of the nervous system in $\mathrm{AD}$.

The lens (equatorial supranuclear cataracts), the retina (loss of ganglion cells, narrowing of venous blood column), macula (volume decrease), the retinal nerve fiber layer (reduction in thickness at the optic nerve head), the optic nerve (widespread axonal degeneration of the retinal ganglion $\mathrm{M}$-cells), the lateral geniculate nucleus (demyelination, amyloid plaques especially in parvocelullar layers), the superior colliculus [amyloid plaque and neurofibrillary tangle (NFT) accumulation], the pulvinar (amyloid plaques and neuritic plaques), the visual cortexpossibly at later stages of the disease-(neuronal loss, amyloid plaques, and NFT, especially in early-onset forms) have been found to be affected in AD (see Tzekov and Mullan, 2013, for an excellent synthesis).

Psychophysical measures have also revealed noteworthy differences between patients with $\mathrm{AD}$ and controls. Visual acuity might be decreased in $\mathrm{AD}$, as a function of disease severity, and/or under low luminance conditions. Color perception-at least in the blue-violet spectrum-is altered in $\mathrm{AD}$, and visual field measures - when possible with $\mathrm{AD}$ patients-have revealed field constriction with deficits being more severe in the inferior part of the visual field (potentially because of the distribution of senile plaques and NFT in the visual cortex; Tzekov and Mullan, 2013). Contrast sensitivity (CS) is also reduced in both patients with $\mathrm{AD}$ and patients with MCI. Clearest evidence of differences in $\mathrm{CS}$ as a function of group (AD, MCI, Cognitive complaints without performance deficits, Controls) has been found in the upper right visual field using frequency-doubling technology, and CS has been regarded as a potential biomarker of AD (Risacher et al., 2013). Finally, both depth (Mendez et al., 1996) and motion (Gilmore et al., 1994; see Fernandez et al., 2013, for an electrophysiological approach) perception abilities are impaired in AD (see also Mandal et al., 2012, for a global picture).

Several studies demonstrated oculomotor processing impairment in $\mathrm{AD}$, even at early stages of the disease or in mild cognitive impairment (Peltsch et al., 2014; Pereira et al., 2014; Molitor et al., 2015). Increased reaction time to trigger saccades, difficulty to inhibit saccadic reflex, and decreased smooth pursuit velocity, acceleration, and accuracy, have been consistently reported (Boxer et al., 2006, 2012; Garbutt et al., 2008; Crawford et al., 2013). Eye movements involved in visual exploration/search are also impaired in AD (Rösler et al., 2000, 2005; Mosimann et al., 2004; Molitor et al., 2015). For instance, when they were supposed to search for a number among 79 letters randomly distributed on a screen, patients with $\mathrm{AD}$ had deficits in target detection and detection time, associated with more fixations and longer fixation duration (Rösler et al., 2000). Mosimann et al. (2004) found that during a clock-reading task, patients with $\mathrm{AD}$ displayed fewer fixations at the end of each clock hand, a significant delay before their first fixation landed inside these regions of interest, longer fixations, and smaller saccade amplitudes.

Not only do the reviewed studies lend support for an impairment of basic visual anatomy, physiology, and behaviors, but they also suggest that higher-level cognition can be influenced by visual impairment in $\mathrm{AD}$.

\section{Interactions between Visual and Higher-level Cognitive Processes}

As rightly noted by Tzekov and Mullan (2013), psychophysical evaluations in $\mathrm{AD}$ should be carried out with caution because of the potential influence of cognitive (and affective) variables-such as compliance with the instructions, assignment comprehension, and memorization, vigilance required during testing-over the measured performances. In the other way round, the numerous changes that affect vision and eye movements in $\mathrm{AD}$ should be taken into account while evaluating 
later and higher-level processes. This should also be the case of language production tasks. If visual acuity (which is the simplest but most controversial psychophysical measure to identify lowlevel visual impairments in AD, see Tzekov and Mullan, 2013, p. 419) is generally controlled for, other visual or visuomotor variables are scarcely taken into account when testing language performances.

This state of affair, which tends to undermine the granted weight of lower-level perceptual-motor processes in cognitive neuropsychological testing, is all the more problematic that previous empirical studies reported clear interferences, not only between visual impairment and Benton's Facial Recognition, or Visual Form Discrimination tests (Kempen et al., 1994), but also between visual impairment and higher-level-cognition evaluation. For instance, Killen et al. (2013) found that visually impaired elderly individuals scored lower than controls in both the vision-dependent items of the Mini-Mental-StateExamination (MMSE) and the Clock drawing test (CDT), but not when vision-independent items of the MMSE and the CDT were proposed. Even if the visual impairments under consideration were rather severe (e.g., macular degeneration, glaucoma), the data suggest that attention should be paid to the control of visual impairment when higher-level cognition is tested. Note that the reduction in macular volume has been reported in $\mathrm{AD}$ and has been found to be related to cognitive performance (as indexed by the MMSE; Iseri et al., 2006).

A recent review of the literature outlined the overlap between cataract and cognitive impairment (Jefferis et al., 2011). Wood et al. (2010) reported impaired performance in older adults across three cognitive tests (the digit symbol substitution test, trail making test A and B, the Stroop color word test) when cataract conditions were simulated.

As another example, by manipulating the stimulus strength of each item of several tests through contrast sensitivity function filtering (i.e., low-degraded, medium-normal, and high-enhanced stimulus-strength conditions), CroninGolomb et al. (2007) demonstrated that the modification of stimulus strength altered performances in several tests in AD. They found that performances in letter identification, word reading, picture naming, and face discrimination decreased more in $\mathrm{AD}$ patients in comparison with healthy elderly in the low-degraded condition. Interestingly, $\mathrm{AD}$ patients improved their performances to a level equal to their healthy counterparts when stimulus strength was enhanced.

\section{References}

Barsalou, L. W. (1999). Perceptual symbol systems. Behav. Brain Sci. 22, 577-660. doi: 10.1017/s0140525x99002149

Boxer, A. L., Garbutt, S., Rankin, K. P., Hellmuth, J., Neuhaus, J., Miller, B. L., et al. (2006). Medial versus lateral frontal lobe contributions to voluntary saccade control as revealed by the study of patients with frontal lobe degeneration. J. Neurosci. 26, 6354-6363. doi: 10.1523/JNEUROSCI.0549-06.2006

Boxer, A. L., Garbutt, S., Seeley, W. W., Jafari, A., Heuer, H. W., Mirsky, J., et al. (2012). Saccade abnormalities in autopsy-confirmed frontotemporal
In this context, and given the extent of visual alteration in $\mathrm{AD}$, much care should be taken when considering language performances, especially if the task requires conceptual production from visual stimulation.

\section{Conclusion: The Need for More Systematic Evaluations of Visual Processes and More Systemic Reasoning in Neuropsychology}

Taken as a whole, data concerning the effects of $\mathrm{AD}$ over visual processes and those demonstrating the influence of visual impairment on higher-level cognition suggest that controlling for visual impairments in patients with $\mathrm{AD}$ could provide critical information to attribute capacity loss to appropriate processing levels. Neuropsychologists know well the strong time constraints that often feature clinical neuropsychological testing. However, when and where possible, some measures should be performed or taken into account (when they are provided by an ophthalmologist) in order to control for any effect of lower-level process (e.g., visual or visuomotor-encoding processes) impairment over higher-level cognitive "functions" (e.g., language production). This is critical when the protocol involves the perception and the interpretation of a visual scenario. Disentangling the language production impairments from other disorders in this kind of settings implies to (i) more thoroughly examine potential differences in visual and eye movement performances between patients and controls through the use of appropriate visual/visuomotor tests, and (ii) consider those performances as covariates in any further group comparison concerning cognitive abilities, namely when the cognitive test requires the processing of visual information. This is needed to better understand and characterize the cascade of alterations associated with $\mathrm{AD}$; especially because $\mathrm{AD}$ patients "are less likely than healthy elderly individuals to report vision problems to their physicians" and that "sensory deficits can be hidden and may masquerade as higher order deficits" (Gilmore et al., 2004).

Beyond the specific case of language, the proposed approach asks basic questions about our conception of cognitive processes. The discussed effects remind us how much cognition is situated, grounded, embodied in specific perceptual (Goldstone and Barsalou, 1998; Barsalou, 1999) and perceptual-motor (Laurent, 2014) systems, which allow recursive processes, conceptual elaboration, and the enaction of what is more classically regarded as modular "cognitive functions."

lobar degeneration and Alzheimer disease. Arch. Neurol. 69, 509-517. doi: 10.1001/archneurol.2011.1021

Crawford, T. J., Higham, S., Mayes, J., Dale, M., Shaunak, S., and Lekwuwa, G. (2013). The role of working memory and attentional disengagement on inhibitory control: effects of aging and Alzheimer's disease. Age 35, 1637-1650. doi: 10.1007/s11357-012-9466-y

Cronin-Golomb, A., Gilmore, G. C., Neargarder, S., Morrison, S. R., and Laudate, T. M. (2007). Enhanced stimulus strength improves visual cognition in aging and Alzheimer's disease. Cortex 43, 952-966. doi: 10.1016/S00109452(08)70693-2 
Drummond, C., Coutinho, G., Fonseca, R. P., Assunção, N., Teldeschi, A., de Oliveira-Souza, R., et al. (2015). Deficits in narrative discourse elicited by visual stimuli are already present in patients with mild cognitive impairment. Front. Aging Neurosci. 7:96. doi: 10.3389/fnagi.2015.00096

Fernandez, R., Monacelli, A., and Duffy, C. J. (2013). Visual motion event related potentials distinguish aging and Alzheimer's disease. J. Alzheimer's Dis. 36, 177-183. doi: 10.3233/JAD-122053

Garbutt, S., Matlin, A., Hellmuth, J., Schenk, A. K., Johnson, J. K., Rosen, H., et al. (2008). Oculomotor function in frontotemporal lobar degeneration, related disorders and Alzheimer's disease. Brain 131(Pt 5), 1268-1281. doi: 10.1093/brain/awn047

Gilmore, G. C., Morrison, S. R., and Groth, K. E. (2004). "Magnocellular deficit hypothesis in Alzheimer's disease," in Vision in Alzheimer's Disease, eds A. Cronin-Golomb and P. R. Hof (Basel: Karger), 173-198.

Gilmore, G. C., Wenk, H., Naylor, L., and Koss, E. (1994). Motion perception and Alzheimer's disease. J. Gerontol. 49, 52-57. doi: 10.1093/geronj/ 49.2.P52

Glenberg, A. M., Havas, D., Becker, R., and Rinck, M. (2005). “Grounding language in bodily states: the case for emotion," in Grounding of Cognition: The Role of Perception and Action in Memory, Language, and Thinking, eds R. Zwaan and D. Pecher (Cambridge: Cambridge University Press), 115-128.

Glenberg, A. M., and Kaschak, M. P. (2002). Grounding language in action. Psychon. Bull. Rev. 9, 558-565. doi: 10.3758/BF03196313

Goldstone, R. L. (1994). Influences of categorization on perceptual discrimination. J. Exp. Psychol. Gen. 123, 178-200. doi: 10.1037/0096-3445. 123.2.178

Goldstone, R. L., and Barsalou, L. (1998). Reuniting perception and conception. Cognition 65, 231-262. doi: 10.1016/S0010-0277(97)00047-4

Greene, J. D. W. (2005). Apraxia, agnosias, and higher visual function abnormalities. J. Neurol. Neurosur. Psychiatry 76, 25-34. doi: 10.1136/jnnp.2005.081885

Harnad, S. (ed.). (1987). Categorical Perception: The Groundwork of Cognition. New York, NY: Cambridge University Press.

Iseri, P. K., Altinas, O., Tokay, T., and Yüksel, N. (2006). Relationship between cognitive impairment and retinal morphological and visual functional abnormalities in Alzheimer disease. J. Neuroophthalmol. 26, 18-24. doi: 10.1097/01.wno.0000204645.56873.26

Jefferis, J. M., Mosimann, U. P., and Clarke, M. P. (2011). Republished review: cataract and cognitive impairment: a review of the literature. Postgrad. Med. J. 87, 636-642. doi: 10.1136/pgmj.2009.165902rep

Kempen, J. H., Krichevsky, M., and Feldman, S. T. (1994). Effect of visual impairment on neuropsychological test performance. J. Clin. Exp. Neuropsychol. 16, 223-231. doi: 10.1080/01688639408402633

Killen, A., Firbank, M. J., Collerton, D., Clarke, M., Jefferis, J. M., Taylor, J.-P., et al. (2013). The assessment of cognition in visually impaired older adults. Age Ageing 42, 98-102. doi: 10.1093/ageing/afs157

Laurent, E. (2014). Multiscale Enaction Model (MEM): the case of complexity and "context-sensitivity" in vision. Front. Psychol. 5:1425. doi: 10.3389/fpsyg.2014.01425
Mandal, P. K., Joshi, J., and Saharan, S. (2012). Visuospatial perception: an emerging biomarker for Alzheimer's disease. J. Alzheimer's Dis. 31, S117-S135. doi: 10.3233/JAD-2012-120901

Mendez, M. F., Cherrier, M. M., and Meadows, R. S. (1996). Depth perception in Alzheimer's disease. Percept. Mot. Skills 83, 987-995. doi: 10.2466/pms.1996.83.3.987

Molitor, R. J., Ko, P. C., and Ally, B. A. (2015). Eye movements in Alzheimer's disease. J. Alzheimer's Dis. 44, 1-12. doi: 10.3233/JAD-141173

Mosimann, U. P., Felblinger, J., Ballinari, P., Hess, C. W., and Müri, R. M. (2004). Visual exploration behaviour during clock reading in Alzheimer's disease. Brain 127(Pt 2), 431-438. doi: 10.1093/brain/awh051

Peltsch, A., Hemraj, A., Garcia, A., and Munoz, D. P. (2014). Saccade deficits in amnestic mild cognitive impairment resemble mild Alzheimer's disease. Eur. J. Neurosci. 39, 2000-2013. doi: 10.1111/ejn.12617

Pereira, M. L., Camargo, M. V., Aprahamian, I., and Forlenza, O. V. (2014). Eye movement analysis and cognitive processing: detecting indicators of conversion to Alzheimer's disease. Neuropsychiatr. Dis. Treat. 10, 1273-1285. doi: 10.2147/NDT.S55371

Pulvermüller, F., and Fadiga, L. (2010). Active perception: sensorimotor circuits as a cortical basis for language. Nat. Rev. Neurosci. 11, 351-360. doi: $10.1038 / \mathrm{nrn} 2811$

Risacher, S. L., WuDunn, D., Pepin, S. M., MaGee, T. R., McDonald, B. C., Flashman, L. A., et al. (2013). Visual contrast sensitivity in AD, MCI, \& older adults with cognitive complaints. Neurobiol. Aging 34, 1133-1144. doi: 10.1016/j.neurobiolaging.2012.08.007

Rosler, A., Mapstone, M., Hays-Wicklund, A., Gitelman, D. R., and Weintraub, S. (2005). The "zoom lens" of focal attention in visual search: changes in aging and Alzheimer's disease. Cortex 41, 512-519. doi: 10.1016/S0010-9452(08)70191-6

Rosler, A., Mapstone, M. E., Hays, A. K., Mesulam, M. M., Rademaker, A., Gitelman, D. R., et al. (2000). Alterations of visual search strategy in Alzheimer's disease and aging. Neuropsychology 14, 398-408. doi: 10.1037/0894-4105.14.3.398

Tzekov, R., and Mullan, M. (2013). Vision function abnormalities in Alzheimer disease. Surv. Ophtamology 59, 414-433. doi: 10.1016/j.survophthal. 2013.10.002

Wood, J., Chaparro, A., Anstey, K., Lacherez, P., Chidgey, A., Eisemann, J., et al. (2010). Simulated visual impairment leads to cognitive slowing in older adults. Optom. Vis. Sci. 87, 1037-1043. doi: 10.1097/OPX.0b013e3181fe64d7

Conflict of Interest Statement: The authors declare that the research was conducted in the absence of any commercial or financial relationships that could be construed as a potential conflict of interest.

Copyright (c) 2015 Laurent and Noiret. This is an open-access article distributed under the terms of the Creative Commons Attribution License (CC BY). The use, distribution or reproduction in other forums is permitted, provided the original author(s) or licensor are credited and that the original publication in this journal is cited, in accordance with accepted academic practice. No use, distribution or reproduction is permitted which does not comply with these terms. 